\title{
CrowdAidRepair: A Crowd-Aided Interactive Data Repairing Method
}

\author{
Jian Zhou ${ }^{\dagger}$, Zhixu $\mathrm{Li}^{\dagger \star}$, Binbin $\mathrm{Gu}^{\dagger}$, Qing $\mathrm{Xie}^{\$}$, Jia Zhu ${ }^{\ddagger}$, \\ Xiangliang Zhang ${ }^{\$}$, and Guoliang $\mathrm{Li}^{£}$ \\ † School of Computer Science and Technology, Soochow University, China \\ $¥$ School of Computer Science, South China Normal University, China \\ $\$$ The King Abdullah University of Science and Technology, Jeddah, Saudi Arabia \\ $£$ Department of Computer Science and Technology, Tsinghua University, China \\ \{jzhou_jz, gu.binbin\}@hotmail.com, zhixuli@suda.edu.cn, jzhu@m.scnu.edu.cn, \\ \{qing.xie, xiangliang.zhang\}@kaust.edu.sa, liguoliang@tsinghua.edu.cn
}

\begin{abstract}
Data repairing aims at discovering and correcting erroneous data in databases. Traditional methods relying on predefined quality rules to detect the conflict between data may fail to choose the right way to fix the detected conflict. Recent efforts turn to use the power of crowd in data repairing, but the crowd power has its own drawbacks such as high human intervention cost and inevitable low efficiency. In this paper, we propose a crowd-aided interactive data repairing method which takes the advantages of both rule-based method and crowd-based method. Particularly, we investigate the interaction between crowd-based repairing and rule-based repairing, and show that by doing crowd-based repairing to a small portion of values, we can greatly improve the repairing quality of the rule-based repairing method. Although we prove that the optimal interaction scheme using the least number of values for crowd-based repairing to maximize the imputation recall is not feasible to be achieved, still, our proposed solution identifies an efficient scheme through investigating the inconsistencies and the dependencies between values in the repairing process. Our empirical study on three data collections demonstrates the high repairing quality of CrowdAidRepair, as well as the efficiency of the generated interaction scheme over baselines.
\end{abstract}

\section{Introduction}

Data repairing aims at discovering and correcting erroneous data in databases. So far, various data repairing solutions have been developed to automatically detect and repair erroneous data in databases [12]. The main stream of rule-based solutions $[2,8,9]$ rely on a variety of quality rules such as FD/CFDs $[1,4,11]$ to detect violations and conflicts between data. By resolving these violations and conflicts, they expect to fix the erroneous data. However, without having the background knowledge, the existing rule-based method just follows some simple modification-strategy (such as minimum-modification) to make modifications $[2$, 9], which as a result, may produce more errors.

\footnotetext{
* The corresponding author
} 
Recent efforts use the power of Crowd for data repairing, which let the crowd to help make right modification decisions according to predefined quality rules. Basically, these crowd-based methods can effectively improve the quality of the data after the repairing. For instance, Yakout et. al. [13] use user's feedback to repair a database and to adaptively refine the training set for a repairing model. However, dislike using rules, no repairing method can solve all the conflicts with one single repairing model. On the other hand, the NADEEF system [3] allows the users to specify data quality rules and how to repair it through writing code that implements predefined classes. However, although some efforts are made, it still requires high labor cost for data repairing. In addition, any methods relying on humans can not be very efficient since humans need to take rest anyway.

In this paper, we propose a novel combined repairing method, CrowdAidRepair, which performs crowd-based repairing and rule-based repairing alternatively for achieving a high repairing quality at the minimum crowd cost. Specifically, we still rely on FD/CFDs to identify conflicts between values, but we do rule-based repairing to a conflict only when this repairing operation can satisfy a predefined quality constraint. When no more conflict can be repaired by the rules, we select some values for crowd-based repairing to let more values be repairable to rule-based method. We continue with this interactive repairing process iteratively until no more values can be modified. To this end, CrowdAidRepair faces a challenge of selecting the least number of values for crowd-based repairing to maximize the number of values for rule-based repairing. Ideally, an optimal interaction scheme minimizes the number of issued crowd-based repairing operations for resolving the detected conflicts correctly.

This scheduling problem for the interaction is nontrivial: Primarily, to reach the minimum crowd cost, we hope to do crowd-based repairing only to those conflicts that can never be resolvable to rule-based repairing. However, we do not know a priori which conflicts can never be resolved correctly by rule-based repairing until all the other conflicts are resolved. Furthermore, the whole interaction issue is considered in a dynamic setting. As more and more conflicts are resolved, the rule-based repairing result to every unresolved conflict might be changed, and the set of unresolved conflicts will also be changed as some new conflicts will be generated while some old ones will be solved/dismissed.

We analyze in theory that the optimal interaction scheme is not feasible to be achieved, and thus we propose our alternative algorithm that can generate an efficient scheme for the interaction between rule-based repairing and crowdbased repairing. In particular, we investigate the inconsistency that each value brings to the database, according to which we estimate a disharmonious score for each value. We will justify that a value with a higher disharmonious score should have a high priority to be checked with the crowd. Besides, although we are in a dynamic setting to schedule conflicts and their covered values for repairing, we can still fix something based on the dependency relations between conflicts and then make decisions accordingly. A challenge is to solve the dependency loop between conflicts and some greedy heuristic algorithm will be proposed to tackle with this NP-hard problem. 


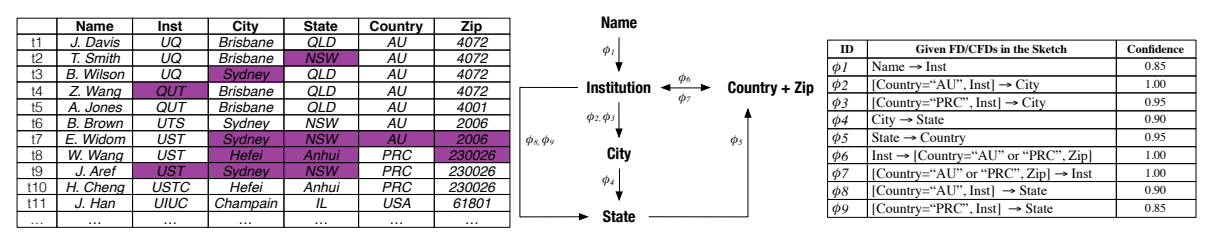

(a) Example Table with 13 Errors Highlighted

(b) A set of the Constraints (FDs/CFDs) holding on the Table

Fig. 1. A Running Example for Illustration

Contributions. We develop CrowdAidRepair, a novel Crowd-Aided Data Repairing approach, which performs crowd-based and rule-based repairing alternatively for achieving a high repairing quality at the minimum crowd cost. We identify and study the quality-constrained interaction problem between crowdbased and rule-based repairing, targeting at a balance between repairing quality and repairing cost. After proving in theory that the optimal interaction scheme is unlikely to be identified, we propose our algorithm to generate efficient interaction schemes.

Roadmap. The rest of the paper is organized as follows: We define the problem in Sec. 2, and then present our algorithm in Sec. 3. The experiments are reported in Sec. 4, followed with related work in Sec. 5. We conclude in Sec. 6.

\section{Preliminary and Problem Statement}

\subsection{Preliminary on Rule-based Repairing}

Definition 1. We say a set of values are correct if all values in this set are correct. We say a Conflict happens between two sets of values if the two sets of values cannot be both correct.

The rule-based repairing method relies on a set of predefined quality rules to detect conflicts between data, and then work to resolve these conflicts with expecting to clean relevant errors that have aroused these conflicts. Particularly in this paper, we take FD/CFD as an example of quality rules to show how our method works. For easier understanding, we present the preliminary with a running example.

Example 1. Given a personal contact data depicted in Fig. 1(a), where each tuple contains the Name, Email and Inst (Institution) of a person, in addition to one's address information: City, State, Country and Zip. We highlight errors waiting to be unveiled and corrected in the table. A set of FD/CFDs holding on the table are listed in Fig. 1(b).

1) Conflicts Detection. According to the given FD/CFDs, a number of conflicts between data can be detected from the table. For example, according to $\phi_{2}$, $t_{1}$ [City] ("Brisbane") and $t_{3}$ [City] ("Sydney") are conflicted with each other as they both correspond to the same Inst ("UQ"). Fig. 2(a) shows that 26 conflicts can be identified according to the constraints in Table 1(a), where each 


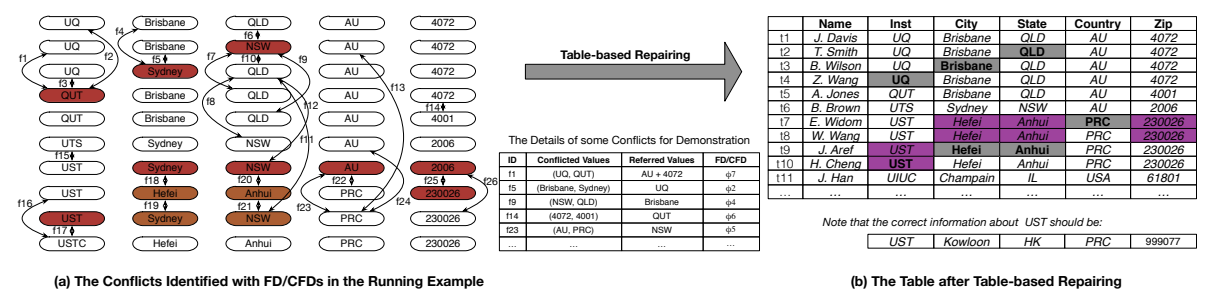

Fig. 2. The Performance of Rule-based Repairing Method

node denotes an attribute value in the table (erroneous values are highlighted), and each line between two nodes denotes a conflict between the two nodes.

2) Conflicts Resolution. When a conflict happens between values, some values should be modified in order to resolve the conflict. In order to resolve all the conflicts in a database, some works tend to make the least changes to the data set $[2,9]$, while others prefer to make the most likely correct changes based on some simple prediction model [8,12]. For example in Fig. 2(a), since $t_{4}$ [Inst] ("QUT") is conflicted with three other values ("UQ"). To resolve the three conflicts, we either change $t_{4}$ [Inst] ("QUT") into "UQ" (cost is 1), or change the three "UQ" into "QUT" (cost is 3). The first modification way is preferred according to either of the two criterions. Fortunately, this is also the correct modification way.

However, the criterions will make wrong decisions in three situations below:

1) It is very likely to make wrong decisions based on a simple criterion. For example, both $t_{8}$ [Inst] ("UST") and $t_{9}$ [Inst] ("UST") are conflicted with $t_{10}$ [Inst] ("USTC"), to make the least change, $t_{10}$ [Inst] ("USTC") will be changed into "UST". As a result, one more error is produced.

2) Some conflict contains no errors, such as the conflict $f_{10}$ between $t_{8}$ [Inst] ("UST") and $t_{10}$ [Inst] ("USTC") in Fig. 2, but the method tends to make corrections to every conflict;

3) The method can not make right corrections when there is no correct correction value to a position within the data set. For instance, in the conflict $f_{13}$ and $f_{14}$, both the two values ("Sydney" and "Hefei") are incorrect City that "UST" locates at (which should be "Kowloon (HK)"), but the method will still pick one from these erroneous values as the correction value.

3) Correction Confidence Estimation. A correction by rule-based repairing is decided jointly by the FD/CFD and all relevant values that used in deducing this correction value. Therefore, the quality of a correction is also determined by the quality of these referred values that are used to deduce this correction value, and the confidence of the referred $\mathrm{FD} / \mathrm{CFD}$, that is,

$$
c\left(v_{c}\right)=c(\phi) \times \prod_{v_{i} \in V_{R}} c\left(v_{i}\right)
$$

, where $V_{R}$ contains a set of referred values that used to deduce $v_{c}$ for the position, $c(v)$ denotes the confidence of a value $v$, and $c(\phi)$ denotes the confidence of $\phi$. 


\subsection{Problem Statement in the Interaction}

We still rely on FD/CFDs to identify conflicts between data, but to identify and correct erroneous values in these conflicts, we consider to involve the crowd into the repairing process to help improve the repairing quality in an efficient interactive way. Particularly, we temporarily neglect the wrong modifications that might be made by the crowd in this paper, and will take it as future work.

The basic interaction can be described as: We set a quality constraint and do rule-based repairing to those conflicts that can satisfy the quality constraint. Then we select some values for crowd-based repairing to let more values be repairable (or so-called deducible) to rule-based method. We continue with this interactive repairing process iteratively until no more values can be modified.

The interaction between crowd-based and rule-based repairing can be represented by a sequence of value sets, denoted as $\mathcal{S}=\left\langle\mathcal{T}_{0}, \mathcal{W}_{1}, \mathcal{T}_{1}, \mathcal{W}_{2}, \mathcal{T}_{2}, \cdots, \mathcal{W}_{n}, \mathcal{T}_{n}\right\rangle$, where $\mathcal{W}_{i}$ is a set of values for repairing at the $i$-th crowd-based repairing step and $\mathcal{T}_{i}$ is a set of values for repairing at the $i$-th rule-based repairing step, $\forall i \neq j, \mathcal{W}_{i} \cap \mathcal{T}_{i}=\mathcal{W}_{i} \cap \mathcal{T}_{j}=\mathcal{W}_{i} \cap \mathcal{W}_{j}=\mathcal{T}_{i} \cap \mathcal{T}_{j}=\emptyset$, and $\forall i, \mathcal{W}_{i} \subseteq \mathbb{V}, \mathcal{T}_{i} \subseteq \mathbb{V}$, where $\mathbb{V}$ denotes the domain of all values in the data set. Note that there is no fix number of values for repairing. An interaction scheme is a qualified one as long as it resolves all the detectable conflicts in the data set.

Since the cost of a crowd-based repairing operation is much more expensive than a rule-based repairing operation or any other computational process, the cost of CrowdAidRepair following an interaction scheme $\mathcal{S}$ can be roughly represented by the number of values for crowd-based repairing in $\mathcal{S}$, i.e., $\operatorname{cost}(\mathcal{S})=\sum_{1 \leq i \leq n}\left|\mathcal{W}_{i}\right|$, where $|\cdot|$ is the size of a set.

Definition 2. (Quality-Constrained Interaction Problem). Given a relational table $T$ for repairing, a set of predefined FD/CFDs $\Phi$ holding on $T$, a quality measuring scheme $c(\cdot)$ and a quality threshold $\tau(0 \leq \tau \leq 1)$, the object is to identify an optimal interaction scheme $\mathcal{S}_{\text {op }}$ for repairing values in $T$, which satisfies: (1) resolving all the conflicts in $T$ w.r.t. $\Phi$; (2) $\forall v_{c}, c\left(v_{c}\right) \geq \tau$, where $v_{c}$ denotes a correction value; (3) $\forall \mathcal{S}^{\prime}$ satisfying the above two conditions, we have $\operatorname{cost}\left(\mathcal{S}_{o p}\right) \leq \operatorname{cost}\left(\mathcal{S}^{\prime}\right)$.

See the situation in the running example, an optimal interaction scheme constructed manually can be:

$<\left\{t_{4}[\text { Inst }]\right\}_{w},\left\{t_{3}[\text { City }], t_{2}[\text { State }]\right\}_{t},\left\{t_{7}[\right.$ Country $], t_{7}[$ Zip $\left.]\right], t_{8}[$ Zip $\left.]\right], t_{9}[$ Inst $\left.]\right\}_{w}$,

$\left\{t_{8}[\right.$ City $\left.]\right], t_{8}[$ State $\left.\left.]\right]\right\}_{t},\left\{t_{7}[\right.$ City $], t_{7}[$ State $\left.\left.]\right]\right\}_{w},\left\{t_{8}[\right.$ City $\left.]\right], t_{8}[$ State $\left.\left.]\right]\right\}_{t}>$, which has 7 values for crowd-based repairing, and the left 6 values for rule-based repairing.

However, the optimal interaction scheme is not feasible to be constructed automatically as described below. Proof to Theorem 1 can be found with the link: http://ada.suda.edu.cn/Uploads/File/201512/04/1449212591654/proof-dasfaa.pdf.

Theorem 1. The optimal interaction scheme to the Quality-Constrained Interaction problem is not feasible to be achieved. 


\section{A Quality-Constrained Interaction Algorithm}

We present our algorithm for generating an efficient interaction scheme. The key problem here lies on how to select values for crowd-based repairing at each crowd-based repairing step.

Initially, we tend to choose the value that has aroused the most conflicts between data for crowd repair, such that the most values will become deducible in the next rule-baed repairing step. In order to find out the value that has aroused the most conflicts between data, we estimate a so-called "disharmonious degree" (or $d$ Score for short) for each value, to denote the "disharmony" between this value and all the other values in the data set. We will introduce how we estimate the dScore of each value in Sec. 3.1.

In addition to dScore, the dependency relations between conflicts should also be taken into account. We say a conflict $f_{a}$ depending on another conflict $f_{b}$, if some values in $f_{b}$ are the reasons (or part of the reasons) that have aroused the conflict in $f_{a}$ according to some FD/CFD. Let a conflict $f_{a}$ depends on another conflict $f_{b}$, we normally should process $f_{b}$ prior to processing $f_{a}$ for three reasons below: (1) Initially, It is possible that after the conflict in $f_{b}$ is resolved, the conflict in $f_{a}$ is dismissed automatically without any repairing operations. (2) To say the least, even if $f_{a}$ is a true conflict and we need to do crowd-based operations to check the values inside it, sometimes we have no other choices but to rely on those values in the conflicts that $f_{a}$ depends on to formulate crowdbased repairing queries. (3) Lastly, after we process all conflicts it depends on, we can update the $d S$ cores for the values in $f_{a}$ for better judging which value is more likely an error.

Although we are in a dynamic setting to schedule conflicts and their covered values for repairing, we can still fix something based on the dependency relations between conflicts and then make decisions accordingly. A challenge is to solve the dependency loop between conflicts. We will discuss this in Sec. 3.2.

\section{1 dScore: Estimating the Incorrectness of Values}

The dScore of a value can be roughly reflected by the number of conflicts it brings to the data set. We first introduce how to calculate the $d S c o r e$ for each value in a simplified case. To begin with, we assume that the data set is consistent without the value at a position, that is, all the other values in the data set appear to be in harmony. Then the value at this position comes, which may bring conflicts in two ways: (1) itself conflicts with some values; (2) it may let some values involved in a conflict. Usually, the more conflicts it brings to the data set, the higher probability it is an erroneous value. In other words, the $d S$ core of a value can be manifested as the number of conflicts it caused in this simple setting.

We now consider the situation in real case, where there are already erroneous values and conflicts in the data set. When a new value at a position comes, either an erroneous one or not, it brings some changes anyway, such as producing new conflicts, or voting for existing conflicts. In this case, the $d S c o r e$ of a value can be manifested by two things: (1) the new conflicts produced, and the "credibility", 
or what we call the $c S c o r e$ of these conflicts, which will be discussed in Eq. (3); (2) the changes on the $c$ Score of existing conflicts. Specifically, $d S \operatorname{Score}(v)$ of a value $v$ can be calculated by:

$$
d \operatorname{Score}(v)=\alpha \times \sum_{f \in F(v)} \Delta(c \operatorname{Score}(f))
$$

where $\alpha$ is a normalization factor to scale $d S \operatorname{core}(v)$ between 0 and $1, F(v)$ contains all conflicts that are influenced by putting $v$ into the data set, and $\Delta(c S c o r e(f))$ is the change on the $c$ Score of a conflict $f$.

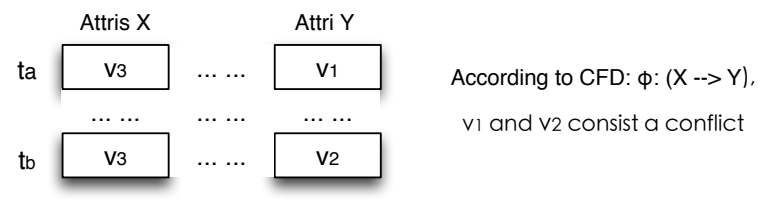

Fig. 3. An Example Conflict with Relevant Values and CFD

In particular, the $c$ Score of a conflict $f$ is decided by four relevant values as given in Fig. 3. Previous work considers that a conflict is consisted of two values such as $v_{1}$ and $v_{2}$ in the figure, but a conflict is also closely related to another two values which are referenced to identify the conflict according to a certain $\mathrm{CFD}$, such as the two $v_{3}$ in the figure. Thus, the correctness of the four values jointly decide the cScore of a conflict $f$. Furthermore, when a conflict is voted as a conflict by several groups of values w.r.t. different CFDs, we only pick the one with the highest $c S c o r e$ as the final $c$ Score of the conflict. More specifically,

$$
c S c o r e(f)=\underset{\phi \subseteq \Phi(f)}{\operatorname{ArgMax}}\left[c(\phi) \times \prod_{v_{i}^{\prime} \in V(f, \phi)}\left(1-d \operatorname{Score}\left(v_{i}^{\prime}\right)\right)\right]
$$

where $\Phi(f)$ is the set of CFDs that voted $f$ as a conflict, and $V(f, \phi)$ contains all values related to the conflict $f$ w.r.t. $\phi$.

\subsection{Employing Dependencies between Conflicts}

We consider the dependencies between conflicts in scheduling conflicts for repairing. We first get the dependency relations among all conflicts, and then build a conflicts dependency graph based on these relations.

1) Relations between Conflicts. Basically, there are three kinds of relationships between each pair of conflicts. The first is the Dependency Relation as we introduced above. Note that the dependency relation is transitive, that is, if $f_{a}$ depends on $f_{b}$, and $f_{b}$ depends on $f_{c}$, then $f_{a}$ also depends on $f_{c}$. Secondly, we say two conflicts are in a Overlapped Relation if they share some positions, such 


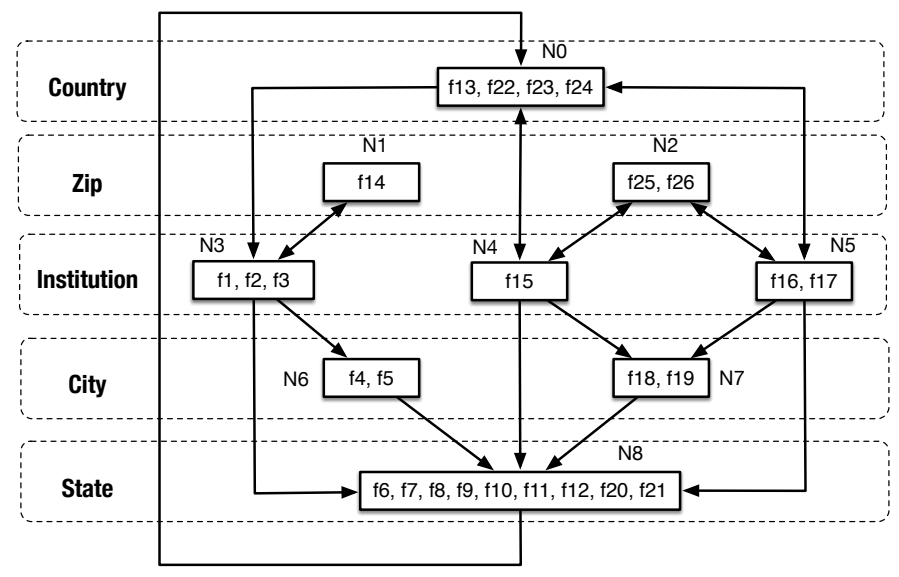

Fig. 4. The Dependency Graph of the Conflicts in Table 1(a)

as $f_{1}$ and $f_{2}$ sharing $t_{4}$ [Inst] ("QUT") in Fig. 2(a). Finally, If two conflicts are in neither of the two relations above, they are Independent from each other.

2) Building Conflicts Dependency Graph. With the relations between all conflicts, we can built a conflicts dependency graph as in Fig. 4 (which is built on Table 1(a)) through the following steps:

(1) Initially, we take each conflict as a node in the dependency graph.

(2) We then put a directed edge pointing from every conflict $f_{a}$ to every other conflict $f_{b}$ if $f_{b}$ depends on $f_{a}$. Note that we only need to put an edge between two conflicts if one directly depends on the other.

(3) Finally, to make the graph easier to process, we merge nodes sharing at least one value into one node (i.e., we put overlapped conflicts into one node), and the directed edges of the same direction between the two nodes are merged into one directed edge.

As introduced above, a conflict should be processed after all the conflicts it depends on are processed. But for those overlapped conflicts in the same node, we need to consider the priority of each value that involved in the conflicts for checking. Here we can still rely on the $d$ Scores of these values. A value with a highest $d S c o r e$ in a node can be checked firstly. Each time a value is modified, the graph needs to be updated accordingly.

\subsection{Tackling Dependency Loops}

The main challenge here is how to schedule those conflicts in dependency loops for processing. We say a number of conflicts are in dependency loop if they depend on each other such as $f_{1}, f_{2}, f_{3}$ and $f_{14}$. In this situation, the dependencybased interaction principle mentioned above dose not work at all. Things become more intractable when there are several loops overlapped with each other at different nodes. As in Fig. 4, there are 19 loops in total and almost every loop is 
overlapped with some other loops at some nodes. Basically, we have to choose one (or more than one) node in a loop to process to "break up" the loop. In order to minimize the cost, we have to be very careful in selecting the break-up node for a loop as different break-up nodes will bring different costs.

Theorem 2. It is an NP-hard problem to break up loops in a dependency graph with the minimum crowd-based repairing cost.

We put the proof to Theorem 2 online. In the following, we give our greedy algorithm to break up loops in a dependency graph.

1) Breaking up a Single Loop. We basically consider two factors in selecting the break-up node for a loop: (1) factor 1: the number of values that must be verified in a node for breaking up the loop (for easier presentation, we call these values as break-up values); (2) factor 2 : the $d S$ cores of these break-up values in a node. Usually, we tend to select the node with the least number of break-up values holding the highest $d S c o r e s$ as the break-up node for the loop. More specifically, we calculate a break-up score, or bScore for short, for each node in a loop as given in Eq. 4 below. Among all nodes in a loop, the node with the highest $b$ Score will be selected as the break-up node in priority.

$$
b \operatorname{Score}(\mathcal{N}, \mathcal{L})=\prod_{v \in V_{b}(\text { node }, \text { loop })} d \operatorname{Score}(v)
$$

where $V_{b}(\mathcal{N}, \mathcal{L})$ is the set of break-up values in node for breaking up loop.

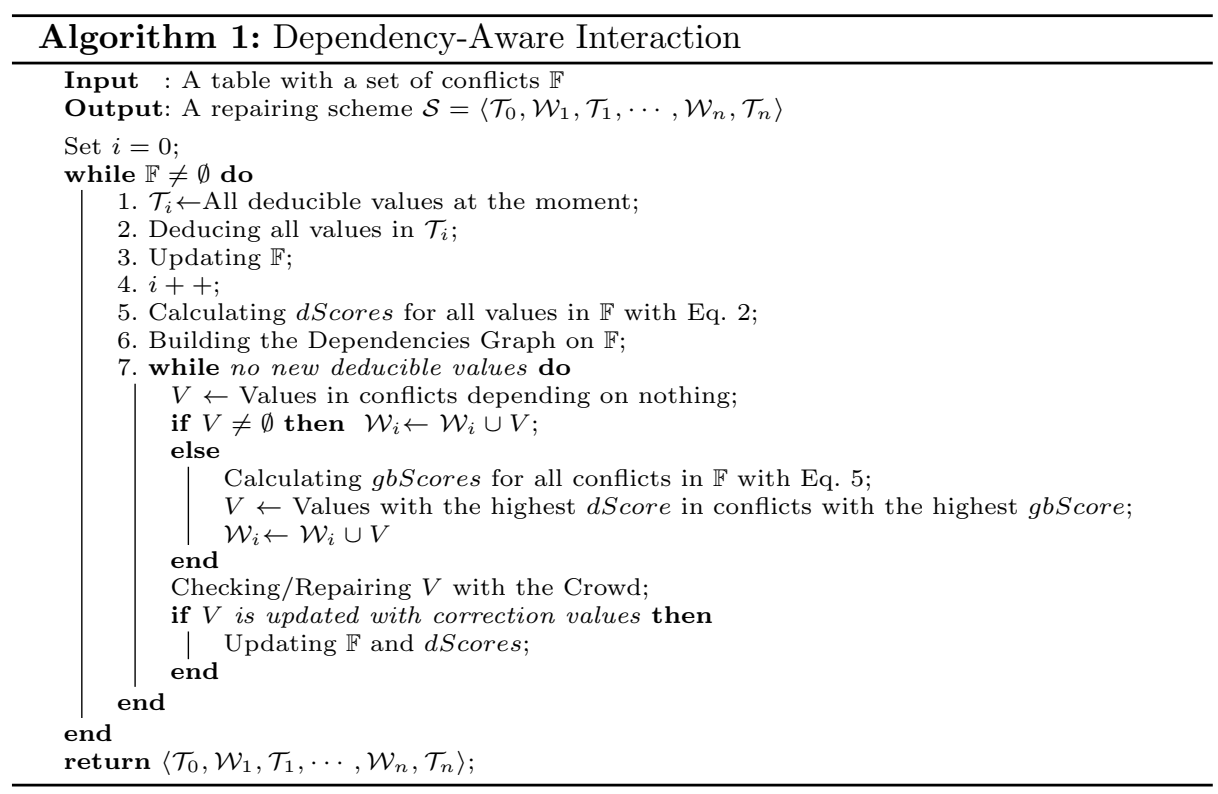


2) Breaking up Multiple Loops. For a number of loops overlapped with each other, we can not simply decide the break-up nodes for a single loop. Otherwise, we may not be able to reach the best performance in minimizing the number of crowd-based operations. For each node, we consider a global bScore, or gbScore for short, to denote its break-up score for all loops in the graph, and the one with the highest gbScore will be selected as the break-up node in priority. The gbScore of a node is decided by two factors: (1) the local bScore of the node in each loop; and (2) the benefit of solving each loop, which is actually the number of values that can be moved out from the loops. More specifically,

$$
\operatorname{gbScore}(\mathcal{N})=\sum_{\mathcal{L} \in L(\mathcal{N})}[b \operatorname{Score}(\mathcal{N}, \mathcal{L}) \times \operatorname{benefit}(\mathcal{N}, \mathcal{L})]
$$

where $L(\mathcal{N})$ is the set of loops having $\mathcal{N}$ as its node in the graph, and the benefit $(\mathcal{N}, \mathcal{L})$ is the benefit of breaking up $\mathcal{L}$ by solving $\mathcal{N}$, which is mainly decided by the number of values in $\mathcal{L}$.

\subsection{Dependency-Aware Interaction Algorithm}

A formal description of this algorithm is given in Alg. 1. Initially, we build the conflicts dependency graph for a data set. For those nodes depending on nothing, we keep on choosing the value with the highest $d$ Score within each node for crowd-based repairing until all the conflicts in the node are resolved. When there is no node of this kind but only loops, we calculate the gbScores for all nodes in these loops, and choose the one with the highest gbScore to process to break up the loops. Each time a value is modified, we need to update the graph and all bScores and gbScores. The algorithm stops when the graph is empty. Basically, the computation complexity of Alg. 1 is $O(m \log m+n)$, where $m$ is the number of nodes which are in the loops of the graph and $n$ is the number of nodes which are not in the loops of the graph.

Example 2. We apply the algorithm to the running example and the interaction scheme generated is depicted in Table 1. Overall, we issue crowd-based operations for 10 values, among which 8 values are true erroneous values while the other 2 values are correct values. Meanwhile, 5 erroneous values are corrected by rulebased repairing.

Theorem 3. The crowd cost of the scheme generated by Algorithm 1 is not larger than $\frac{e-1}{e}$ times the cost of the optimal interaction scheme.

We also put the proof to Theorem 3 online.

\section{Experiments}

We perform our experiments on two real and one synthetic data sets. We also employ 20 users in our research group to act as crowd, all of whom have known part of the ground-truth knowledge a priori. 
Table 1. The Interaction Scheme generated by Alg. 1

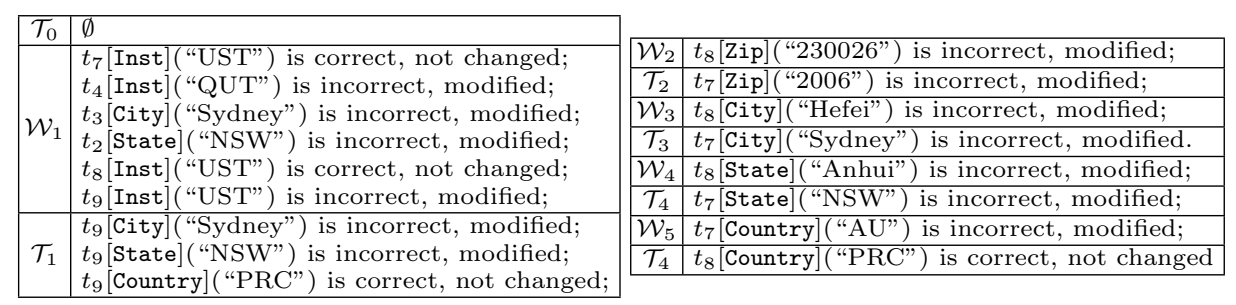

1) Personal Information Table (PersonInfo): This is a 50k-tuples, 9-attributes table, which contains contact information for academics including name, email, title, university, street, city, state, country and zip code.

2) DBLP Publication Table (DBLP): This is a 100k-tuples, 5-attributes table. Each tuple contains information about a published paper, including its title, first author and his/her affiliation, conference name, year and venue.

3) Synthetic Table (Syn): We also generate a 1million-tuples, 100-attributes table following a scheme containing 100 randomly generated approximate attribute dependencies with confidences near-uniformly distributed between 0.7 and 1, where the first attribute is the key attribute.

All the three data sets are relational tables without erroneous data. To generate tables with errors for the experiments, we keep the key attribute value in each tuple and replace non-key attribute values at random positions with attribute values selected from random picked tuples of the table.

\subsection{Repairing Quality Evaluation}

In the following experiments, we compare the repairing quality of PureCrowdRepair (Pure crowd-based Repairing) and CrowdAidRepair with four state-ofthe-art general textual data repairing approaches on the three data sets.

1) Rule-based Most-Likely (CFD-ML): This approach relies on FD/CFDs to detect and correct erroneous data [2], and follows the most-likely correct modification criterion as introduced in Sec. 2.1.

2) Model-based 1 (ERACER): This is a model-based repairing based on belief propagation and relational dependency networks [10]. In contrast to prior work that cleans tuples in isolation, this approach exploits the graphical structure of the data to propagate inferences throughout the database [10].

3) Model-based 2 (SCARE): This is another model-based repairing approach based on maximizing the correctness likelihood of replacement data given the data distribution, which is modelled using statistical machine learning techniques [12].

4) Crowd-based (GuidedRepair): We implement the state-of-the-art crowdbased repairing method proposed in [13], which collects feedbacks from users to adaptively refine the training set for a repairing model. 


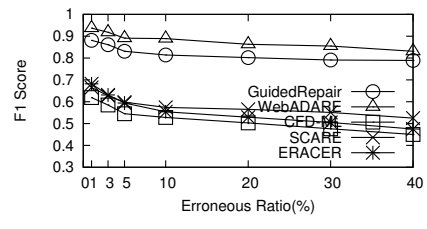

(a) PersonInfo

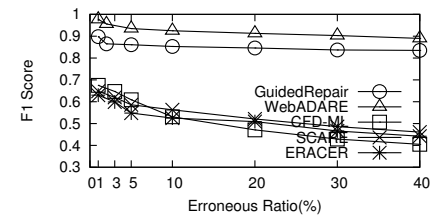

(b) DBLP

Fig. 5. Comparing the F1 Scores of all Methods

Table 2. Comparing the Repairing Quality with Previous Methods

\begin{tabular}{|c||c|c|c||c|c|c|}
\hline \multicolumn{1}{|c||}{} & \multicolumn{3}{c||}{ PersonInfo } & \multicolumn{3}{c|}{ DBLP } \\
\hline & Prec & Recall & F1 & Prec & Recall & F1 \\
\hline CFD-ML & 0.535 & 0.521 & 0.528 & 0.645 & 0.451 & 0.531 \\
\hline ERACER & 0.638 & 0.489 & 0.554 & 0.698 & 0.421 & 0.525 \\
\hline SCARE & 0.655 & 0.512 & 0.574 & 0.743 & 0.453 & 0.563 \\
\hline GuidedRepair & $\mathbf{0 . 8 2 5}$ & $\mathbf{0 . 8 0 4}$ & $\mathbf{0 . 8 1 4} ! !$ & $\mathbf{0 . 8 7 1}$ & $\mathbf{0 . 8 3 7}$ & $\mathbf{0 . 8 5 3 5 ! !}$ \\
\hline PureCrowdRepair & $\mathbf{0 . 9 3 2}$ & $\mathbf{0 . 8 9 1}$ & $\mathbf{0 . 9 1 1}$ & $\mathbf{0 . 9 7 5}$ & $\mathbf{0 . 9 1 1}$ & $\mathbf{0 . 9 4 2}$ \\
\hline CrowdAidRepair & $\mathbf{0 . 9 0 3}$ & $\mathbf{0 . 8 7 6}$ & $\mathbf{0 . 8 8 9}$ & $\mathbf{0 . 9 5 8}$ & $\mathbf{0 . 8 9 5}$ & $\mathbf{0 . 9 2 5}$ \\
\hline
\end{tabular}

We first make a comprehensive comparison on the Precision, Recall and F1 of all the methods at an erroneous ratio of $10 \%$ on the two real data sets. The parameter setting for each method lets the method reaches the best repairing quality (w.r.t. F1). As shown in Table 2, the precision and recall of the rule-based method (CFD-ML) are not high, as it can only make correct modifications to about half of the erroneous values in the data sets, and in $40-60 \%$ chances it makes wrong corrections. Comparatively, the precision of the two model-based methods (ERACER and SCARE) is a bit (5-10\%) higher than the rule-based methods since the models they build can understand the correlation between data and thus make better judgements. On the other hand, their recall is as low as that of the rule-based method, since there are some non-quantitive attributes like email, street, author, and venue which can not be handled well by models.

Apparently, the precision and recall of GuidedRepair, PureCrowdRepair and CrowdAidRepair are much higher $(85+\%$ precision and $85+\%$ recall $)$ than the rule-based and model-based methods. In particular, PureCrowdRepair reaches the highest precision and recall as it lets the crowd to do every correction. The precision and recall of our method (CrowdAidRepair) is a bit less than the PureCrowdRepair method, but higher than the GuidedRepair method. This is because our method takes the advantages of both rules and crowd. which can work better than a model, even if the model is refined by the crowd.

We then compare the F1 scores of all methods at various erroneous ratios $(1 \%, 3 \%, 5 \%, 10 \%, 20 \%, 30 \%, 40 \%)$ by setting $\tau=0.7$ over the two real-world data sets. As demonstrated in Fig. 5, CrowdAidRepair always reaches higher F1 scores than all the other four methods including the GuidedRepair method at various erroneous ratio, which also proves the advantage of CrowdAidRepair over the four other methods. 


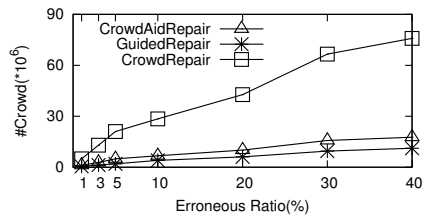

(a) PersonInfo (\#Crowd)

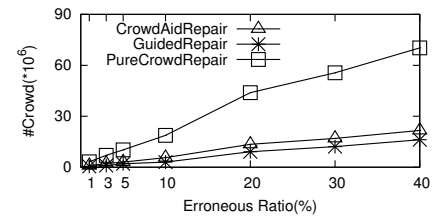

(b) DBLP (\#Crowd)

Fig. 6. Comparing \#Crowd on the Real Data $\operatorname{Sets}(\tau=0.7$, erroneous ratio $=10 \%)$

\subsection{Repairing Cost Evaluation for Methods Using the Crowd}

In this paper, we denote the number of values for crowd repair (\#Crowd) as the crowd cost of a repairing method. We now compare the crowd cost of CrowdAidRepair with PureCrowdRepair and GuidedRepair on \#Crowd.

As demonstrated in Figure 6(c)(d), CrowdAidRepair only uses about $20 \%$ crowd cost of that used by PureCrowdRepair and thus greatly reduces the overhead of the repairing process. However, compared with GuidedRepair, we use a bit higher overhead for reaching a higher repairing quality.

\subsection{Interaction Schemes Evaluation}

To further evaluate the effectiveness of CrowdAidRepair, we compare the effectiveness of two interaction schemes generated by (1) an algorithm relying on dScores only to find the interaction scheme (so called dScore-based scheme); and (2) the CrowdAidRepair algorithm that considers both dScores and conflict dependencies (so called depend-aware scheme). We set the erroneous ratio to $10 \%$, and then compare the repairing quality (precision and recall) and cost of the interactive repairing following each interaction scheme by changing the quality threshold $\tau$ from 0 to 1 .

As shown in Fig. 7(a)(b),the dScore-based scheme and the depend-aware scheme can reach almost the same precision and recall. On the other hand, as shown in Fig. 7(c), the cost of both schemes increases as $\tau$ increases from 0 to about 0.8 , but decreases sharply as $\tau$ increases from 0.8 to 1.0. This makes sense since when the quality constraint becomes too strict, much less values can be repaired to satisfy the constraint. Nonetheless, the cost of the depend-aware interaction scheme is always about $40 \%$ less than the dScore-based scheme, which proves the advantage of the depend-aware scheme over the other scheme.

\section{Related Work}

Data repairing aims at discovering and correcting erroneous data in databases. So far, various data repairing solutions have been developed to automatically detect and repair erroneous data in databases [12]. All existing solutions can be roughly put into three categories below.

The traditional category of methods relies on a variety of constraints including FDs [1,11], CFDs [4], Integrity Constraints [9] and Inclusion Dependencies 


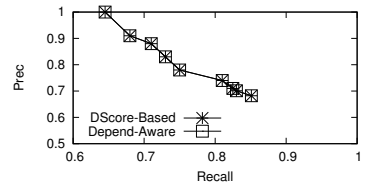

(a) PR (PersonInfo)

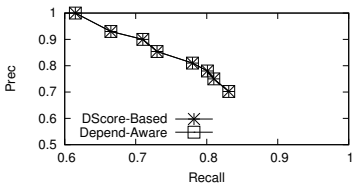

(b) PR (DBLP)

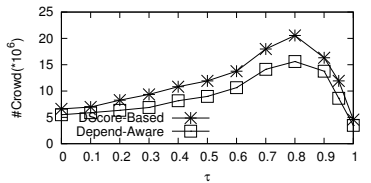

(c) \#Queries (Syn)

Fig. 7. Comparing the Schemes: Precision and Recall in Subfigures (a)(b) (on the two real data set), and \#Queries in Subfigures (c) (Syn data set)

(INCs) [1] to detect inconsistency (or conflicts) between data aroused by erroneous data, and then work on resolving all the conflicts with expecting to fix all erroneous data in this way $[2,8,9]$. For general textual databases, most work in this category use FD/CFDs for repairing as they are the constraints within a single relational table, while some other work uses INCs for repairing between multiple relational tables. Usually, this category of methods can effectively detect a large percent of erroneous data involved in the identified conflicts in a wide range of databases, but to repair these errors and resolve the conflicts, some work tends to make the least changes to the data set $[2,9]$, while others prefer to make the most likely correct changes based on some simple prediction model $[8,12]$. However, neither criterion can have all errors modified correctly.

The second category of solutions are model-based repairing, which usually build some prediction models for detecting and correcting erroneous values in a data set $[6,7,10,12,14,15]$. The construction of the model employs statistical Machine Learning (ML) techniques for data cleaning, which can effectively capture the dependencies and correlations between data in the dataset based on various analytic, predictive or computational models $[12,15]$. However, not every erroneous data can be identified and corrected in the right way since there are always outliers that do not obey the captured constraints.

The third category of solutions are external source based repairing approaches, which leverage the information in reference master data set [5] or user's interaction data such as GuidedRepair [13] and NADEEF [3] for better data cleaning performance. However, the required external information is not always available and thus the methods can not be applied in general scenarios. In this paper, we propose CrowdAidRepair, which is a hybrid repairing approach using the crowd.

\section{Conclusions and Future Work}

We propose CrowdAidRepair, a novel crowd-aided data repairing approach that can greatly enhance the repairing quality of the existing rule-based repairing method with the Crowd help. Extensive experimental results based on several data collections demonstrate that the generated interaction scheme decreases on average $60 \%$ cost of a baseline, and reaches almost the same high repairing quality that was reached by a pure crowd-based retrieving approach. Future work may consider combining CrowdAidRepair with state-of-the-art model- 
based methods, and apply CrowdAidRepair to databases with both incorrect values and missing values.

Acknowledgements: This research is partially supported by Natural Science Foundation of China (Grant No. 61303019, 61402313, 61472263, 61572336), Postdoctoral scientific research funding of Jiangsu Province (No. 1501090B) National 58 batch of postdoctoral funding (No. 2015M581859) and Collaborative Innovation Center of Novel Software Technology and Industrialization, Jiangsu, China.

\section{References}

1. P. Bohannon, W. Fan, M. Flaster, and R. Rastogi. A cost-based model and effective heuristic for repairing constraints by value modification. In SIGMOD, pages 143$154,2005$.

2. G. Cong, W. Fan, F. Geerts, X. Jia, and S. Ma. Improving data quality: Consistency and accuracy. In $P V L D B$, pages 315-326, 2007.

3. M. Dallachiesa, A. Ebaid, A. Eldawy, A. Elmagarmid, I. F. Ilyas, M. Ouzzani, and N. Tang. Nadeef: a commodity data cleaning system. In SIGMOD, pages 541-552, 2013.

4. W. Fan, F. Geerts, X. Jia, and A. Kementsietsidis. Conditional functional dependencies for capturing data inconsistencies. ACM Transactions on Database Systems (TODS), 33(2):6, 2008.

5. W. Fan, J. Li, S. Ma, N. Tang, and W. Yu. Towards certain fixes with editing rules and master data. PVLDB, 3(1-2):173-184, 2010.

6. W. Hua, Z. Wang, H. Wang, K. Zheng, and X. Zhou. Short text understanding through lexical-semantic analysis. International Conference on Data Engineering (ICDE), 2015.

7. J. L. Koh, M. L. Lee, W. Hsu, and K. T. Lam. Correlation-based detection of attribute outliers. In Advances in Databases: Concepts, Systems and Applications, pages 164-175. Springer, 2007.

8. S. Kolahi and L. V. Lakshmanan. On approximating optimum repairs for functional dependency violations. In ICDT, pages 53-62, 2009.

9. A. Lopatenko and L. Bravo. Efficient approximation algorithms for repairing inconsistent databases. In ICDE, pages 216-225, 2007.

10. C. Mayfield, J. Neville, and S. Prabhakar. Eracer: a database approach for statistical inference and data cleaning. In SIGMOD, pages 75-86, 2010.

11. J. Wijsen. Database repairing using updates. ACM Transactions on Database Systems (TODS), 30(3):722-768, 2005.

12. M. Yakout, L. Berti-Équille, and A. K. Elmagarmid. Don't be scared: use scalable automatic repairing with maximal likelihood and bounded changes. In SIGMOD, pages 553-564, 2013.

13. M. Yakout, A. K. Elmagarmid, J. Neville, M. Ouzzani, and I. F. Ilyas. Guided data repair. PVLDB, 4(5):279-289, 2011.

14. B. Zheng, N. J. Yuan, K. Zheng, X. Xie, S. Sadiq, and X. Zhou. Approximate keyword search in semantic trajectory database. In Data Engineering (ICDE), 2015 IEEE 31st International Conference on, pages 975-986. IEEE, 2015.

15. X. Zhu and X. Wu. Class noise vs. attribute noise: A quantitative study. Artificial Intelligence Review, 22(3):177-210, 2004. 